\title{
SEROEPIDEMIOLOGÍA DE HEMBRAS BOVINAS NATURALMENTE INFECTADAS POR Neospora caninum EN CORDOBA, COLOMBIA
}

\section{SEROEPIDEMIOLOGY OF FEMALE COWS NATURALLY INFECTED WITH Neospora caninum IN CORDOBA, COLOMBIA}

\begin{abstract}
José A. Cardona ${ }^{1}$, Yordan Martínez ${ }^{2}$, César A. Betancur ${ }^{3}$
${ }^{1}$ MVZ, Esp, M.Sc, Ph.D., profesor titular Medicina y Clínica de Grandes Animales, Semillero de Estudios e Investigaciones en Medicina de Grandes Animales, Grupo de Investigación MEGA, Departamento de Ciencias Pecuarias. Universidad de Córdoba, AA 354, Montería, Colombia, e-mail: cardonalvarez@hotmail.com; ${ }^{2}$ MVZ. Ph.D., Centro de Estudios de Producción Animal, Facultad de Medicina Veterinaria. Universidad de Granma, Aptado, 21 Granma. Bayamo CP: 85100, Cuba, e-mail: ymartineza@udg.co.cu; ${ }^{3}$ MVZ, M.Sc., profesor asociado de Fisiología y Enfermedades Infecciosas, Semillero de Estudios e Investigaciones en Fisiología Animal Fisiolab. Universidad de Córdoba, Departamento de Ciencias Pecuarias, AA 354, Montería, Colombia, e-mail: cbetanci@yahoo.com
\end{abstract}

Rev. U.D.C.A Act. \& Div. Cient. 18(2): 401-408, Julio-Diciembre, 2015

\section{RESUMEN}

El objetivo del trabajo fue contribuir al conocimiento de la seroepidemiología de Neospora caninum y determinar los niveles de sarcocystina en bovinos, con alteraciones reproductivas. Se evaluaron 162 sueros de bovinos y 28 sueros de caninos, procedentes de 28 fincas, localizadas en el municipio de Montería, mediante ELISA. Se realizó un análisis descriptivo de la información obtenida de cada animal; los resultados, se interpretaron de acuerdo a las variables localización del predio, edad, tipo de sistema productivo, historia de abortos o repetición de servicios, sexo, presencia de caninos y niveles de sarcocystina. Para el cálculo del tamaño de muestras, se utilizó el programa EPIDAT 3.1 y para el análisis de los datos, el test de Chi cuadrado, utilizando el paquete estadístico SPSS versión 16 para Windows de 2008. La seroprevalencia para N. caninum obtenida fue de 74,7\%; no se presentó asociación estadística entre las diversas variables estudiadas y la seropositividad al protozoario ( $p>0,05)$. Se confirma la evidencia de circulación antigénica de $N$. caninum en las ganaderías bovinas de Montería, Córdoba (Colombia), por lo que se hace necesario implementar programas de control y de prevención de diseminación de la infección.

Palabras clave: Neospora caninum, reproducción, seroepidemiología, neosporosis.

\section{SUMMARY}

The aim of the work was to contribute to the knowlege of the seroepidemiology of Neospora caninum and to evaluate sarcocystine levels in animals with reproductive failures in Montería, Córdoba, Colombia. A total of 162 serum samples of cows and 28 canines belonging to 28 farms distributed in the municipality of Monteria were evaluated using the indirect ELISA test to determine $N$. caninum seropositivity. A program EPIDAT 3.1 was carried out to calculate the size of the samples and the data were processed through a chi Square test using SPSS 19 program, results were interpreted according to the variables: zone, age, type of exploitation, and the event or reproductive problem detected, sex, presence of canines and sarcocystina levels. The estimated prevalence of $N$. caninum in cows was $74.7 \%$. No significant associations $(\mathrm{P}>0.05)$ were observed between the variables studied and seropositivity to protozoan. The results obtained to confirm the evidence of the circulation antigenic of $N$. caninum again in the cattle from Montería, Córdoba (Colombia) which it is necessary to implement a control program and prevention of dissemination of infection.

Key words: Neospora caninum, reproduction, seroepidemiology, neosporosis.

\section{INTRODUCCIÓN}

La neosporosis bovina es una enfermedad emergente que causa pérdidas económicas a las ganaderías, debido al 
impacto negativo sobre el desempeño reproductivo de los rebaños, produciendo abortos, mortalidad neonatal y el nacimiento de crías con deficiencias neuromusculares, en bovinos en todo el mundo (Dubey et al. 2006). Es causada por Neospora caninum, un protozoo intracelular obligado, perteneciente a la familia Sarcocystidae, phylum Apicomplexa, estrechamente relacionado con Toxoplasma gondii (Dubey, 2003).

La diferencia en el manejo de ganado de leche y ganado de carne puede explicar la alta prevalencia de neosporosis, en el primero, como consecuencia del recrudecimiento de la infección latente, debido a la inmunodepresión generada por la gestación, el manejo y la lactación (Moore, 2004).

Las rutas de transmisión de $N$. caninum en el ganado incluyen la transmisión vertical y la transmisión horizontal (Trees \& Williams, 2005; Ortega-Mora et al. 2006). La primera es reconocida como la principal ruta para mantener la infección en el ganado, por lo que los becerros son infectados vía transparentaría en madres, crónicamente infectadas (Dubey et al. 2006; 2007). La transmisión horizontal ha sido asociada con la presencia de perros en las fincas, ya que el perro y el coyote han sido descritos como huéspedes definitivos de $N$. caninum, donde el estado sexual del protozoario se desarrolla en el intestino, formando ooquistes, que se eliminan en las heces y, con ello, contaminar agua de bebida y alimentos (Dubey et al. 2007); sin embargo, la neosporosis es definida como una enfermedad parasitaria, que afecta caninos, bovinos, ovinos, caprinos, búfalos, ciervos y equinos, causada por N. caninum (Dubey, 1999a). Equinos, caprinos, ovinos, ciervos, búfalos e, inclusive caninos, se pueden comportar como hospederos intermediarios (Dubey, 1999b).

Al parecer existe relación entre bovinos y perros criados en los establos (Del Campo et al. 2003), siendo mayor la infección en caninos de las zonas rurales, que aquella descrita para perros, en áreas urbanas, debido a la mayor excreción de ooquistes en las heces, por lo que el riesgo de contaminar el agua de bebida y alimentos, es mayor (Moore et al. 2001).

Aunque Dubey et al. (2006) consideran que la transmisión sexual de la enfermedad tiene muy poca importancia, el rol epidemiológico de los toros en la neosporosis bovina es desconocido y su riesgo de transmisión debe ser investigado.

Los quistes parasitarios de $N$. caninum durante la infección, que se desarrollan en el hospedero intermediario varían considerablemente, dependiendo de la especie, de los hospederos y de la edad de los quistes. El quiste consiste en un cuerpo cilíndrico alongado o fusiforme y con apariencia hialina, que reciben el nombre de túbulos de Miescher y se encuentran encerrados por una membrana, que contiene millares de esporas redondeadas, ovales o en forma de hoz, conocidas como cuerpos de Rainey, que están suspendidos en un líquido compuesto, principalmente, por toxina del parásito, llamada sarcocystina, que es liberada al romperse el quiste.

Esta toxina presenta una actividad neurotóxica, a nivel de músculo cardiaco y tejido nervioso, y altera las membranas celulares, mostrando una excesiva captación de agua y liberando mediadores inflamatorios, que producen hipertermia, pudiendo ser letal, además de causar desórdenes generales en los animales afectados y que puede conllevar a pérdidas en la explotación ganadera, por la disminución en la producción o productividad (Azumendi et al. 1995)

En Suramérica, la enfermedad ha sido diagnosticada en países, como Argentina (Campero et al. 1998; Venturini et al. 1999; Moore et al. 2002; 2003), Brasil (Guedes et al. 2008; Moura et al. 2012), Chile (Patitucci et al. 2000), Perú (Silva et al. 2002; Granados et al. 2014), Venezuela (García \& Lista, 2005; Obando et al. 2010; Suárez \& Maldonado, 2012) y Colombia (Zambrano et al. 2001; López et al. 2007; Oviedo et al. 2007).

El objetivo de este estudio fue establecer la seroprevalencia de $N$. caninum en bovinos naturalmente infectados, con alteraciones reproductivas, determinar si existe asociación entre la seroreactividad con la edad, tipo de explotación, sexo y niveles de sarcocystina y si existen otros factores de riesgo, como presencia de perros en las fincas.

\section{MATERIALES Y MÉTODOS}

El estudio fue realizado en el departamento de Córdoba, Colombia, ubicado entre las coordenadas $7^{\circ} 23^{\prime}$ y $9^{\circ} 26^{\prime}$ de latitud Norte y los $74^{\circ} 52^{\prime}$ y $76^{\circ} 32^{\prime}$ de longitud Oeste del meridiano de Greenwich, a una altura de $30 \mathrm{msnm}$, con temperatura promedio anual de $28^{\circ} \mathrm{C}$, humedad relativa del $82 \%$, precipitación media anual de $1.400 \mathrm{~mm}$ y pertenece a la formación climática de bosque tropical lluvioso. Se presentan dos estaciones bien definidas (época de lluvia y época seca) (Pabón et al. 2001).

La investigación propuesta correspondió a un estudio epidemiológico de tipo descriptivo transversal. Para el cálculo del tamaño de muestras, se utilizó el programa EPIDAT 3.1, asumiendo una frecuencia esperada de seropositivos del $12 \%$, un error del $5 \%$, un nivel de confianza del $95 \%$ y una población infinita. El tamaño de la muestra fue determinado por un muestreo al azar, basado en la población bovina de 178.320 hembras bovinas, entre dos y siete años, existente al momento del estudio (ICA-Fedegan, 2012), con peso promedio de $380 \pm 50 \mathrm{~kg}$ y buena condición corporal. En total, se obtuvieron 162 muestras de sangre de bovinos, 
siendo 28 toros y 134 vacas y 28 caninos, provenientes de 28 fincas, incluidas, donde el $32,1 \%$ están dedicadas a la producción de ganado de carne y las restantes $67,9 \%$ fueron sistemas productivos de doble propósito, que estuvieron localizadas en los cuatro puntos cardinales del municipio de Montería, Córdoba (Colombia).

Para la selección de los predios, se tuvo en cuenta la historia de alteraciones reproductivas en hembras bovinas de doble propósito, entre dos y siete años de edad, evidenciadas en abortos y en repetición de servicios, tomando como parámetro, más de tres servicios sin gestación; en todos los casos, se obtuvo el consentimiento informado de los propietarios o administradores para colectar las muestras de sangre de los animales. En cada finca, se colectó muestra de entre cuatro y cinco hembras bovinas, un toro y un canino. En el caso de las hembras, se obtuvo información referente a identificación del animal, edad, raza, tipo de explotación y estado reproductivo.

Previa desinfección del área y sujeción adecuada del animal, se obtuvieron $10 \mathrm{~mL}$ de sangre por punción de la vena coccígea media, en bovinos y cefálica, en caninos, con agujas de 22G y tubos tipo vacutainer sin anticoagulante (Becton Dickinson and Company, Colombia); estas muestras fueron centrifugadas (1.500 rpm durante cinco minutos) (Combi 408, Hanil, Japón), con el fin de obtener el suero y almacenadas en nevera, a $-20^{\circ} \mathrm{C}$. Las muestras fueron procesadas en el laboratorio veterinario FUNCEP de Bogotá, mediante la técnica de ELISA, utilizando el kit anti-Neospora, de laboratorios Idexx Herdchek (Westbrook, USA).

A cada muestra de sangre, se le determinó, también mediante la técnica de ELISA, la concentración de sarcocystina, expresada en UI/L (Pulido et al. 2009). El resultado fue interpretado por el desarrollo de un color azul, el cual, fue observado en un fotómetro (Genesys 10S UV VIS, China), a una densidad óptica de 450nm y su valoración, se hizo teniendo en cuenta los cocientes $\mathrm{S} / \mathrm{P}$ (suero muestra positivo), donde los valores menores que 0,50 , se clasificaron como negativos y los iguales o mayores a 0,50 , como positivos (Pulido et al. 2009).

Para el análisis de los datos, se utilizó el coeficiente de contingencia de Pearson, que utiliza la distribución $\mathrm{X}^{2}$, para determinar posibles asociaciones. Para la obtención de resultados de las distintas variables, se utilizó el paquete estadístico SPSS versión 19 (SPSS Inc, 2007).

Los animales no se sometieron a dolor o estrés innecesario, por lo que fueron inmovilizados, tomando en cuenta las normas técnicas en el manejo y sujeción de animales, enmarcado en el cumplimiento de la Declaración Universal de los Derechos de los Animales, referente a los principios éticos internacionales para la investigación biomédica con animales del CIOMS (Council for International Organizations of Medical Sciences), establecida por la UNESCO (United Nations Educational, Scientific and Cultural Organization) y la OMS (Organización Mundial de la Salud), en 1949 y de la Ley 84 de Octubre 27 de 1989 (Estatuto Colombiano de Protección Animal) (Mrad, 2006).

\section{RESULTADOS Y DISCUSIÓN}

La prevalencia para $N$. caninum, en el municipio de Montería, fue de 74,7\%. Es así, como Zambrano et al. (2001) reportaron la presencia de anticuerpos contra $N$. caninum, en $54,1 \%$ de vacas, con antecedentes abortivos, procedentes de la sabana de Bogotá, de Nariño y de algunas fincas de clima cálido. Del mismo modo, estudios realizados en vacas con antecedentes de trastornos reproductivos en Montería, demostraron la presencia de anticuerpos compatibles, en el 10,2\% de los animales estudiados (Oviedo et al. 2007); asimismo, López et al. (2007) evidenciaron la presencia de $N$. caninum, en el $34,6 \%$ de los bovinos muestreados en el municipio de Fredonia, Antioquia. Esta diferencia podría ser explicada por una exposición, a través de los años, al contacto con animales infectados y que se podría considerar como un buen indicador de exposición natural de los bovinos, a $N$. caninum (Davison et al. 1999).

El patrón endémico es la forma más frecuente de los abortos causados por neosporosis y se presenta en hatos, donde el parásito se transmite, principalmente, de modo vertical. En los rebaños con aborto endémico, se ha observado una marcada correlación entre la seropositividad de las madres y la progenie, con una distribución igual de los animales seropositivos, en los diferentes grupos de edad (Dijkstra et al. 2001; Schares et al. 2002), mientras que el patrón de aborto epidémico, se ha asociado con una infección reciente y la transmisión postnatal del parásito, evidenciado por la falta de asociación entre la seropositividad de las madres y la descendencia y la presencia de IgG anti- $N$. caninum de baja avidez en los animales abortados (Wouda et al. 1999a; McAllister et al. 2000).

Con respecto a la ubicación de las fincas en los cuatro puntos cardinales del municipio, se puede observar en la tabla 1, que hubo una menor frecuencia de animales seropositivos en las fincas localizadas en la zona norte $(63,41 \%)$ versus oriente $(80,49 \%)$, sur $(77,50 \%)$ y occidente $(78,05 \%)$; sin embargo, no hubo diferencias estadísticas significativas ( $>>0.05)$, en la frecuencia de seropositividad y la zona geográfica.

La menor frecuencia de animales seropositivos en las fincas localizadas en la zona norte $(63,41 \%)$ puede ser explicada por la menor movilización de ganado en esta área. Las zonas evaluadas en el presente estudio no tuvieron 
Tabla 1. Seropositividad a N. caninum y nivel de sarcocystina en bovinos, de cuatro zonas del municipio de Montería.

\begin{tabular}{|c|c|c|c|c|}
\hline & \multicolumn{4}{|c|}{ Ubicación } \\
\hline ítems & Norte & Este & Sur & Oeste \\
\hline Seroprevalencia (\%) (B) & 63,41 & 80,49 & 77,50 & 78,05 \\
& $(34 / 162)$ & $(35 / 162)$ & $(52 / 162)$ & $(41 / 162)$ \\
\hline Nivel de sarcocystina (B) UI/L & 127,37 & 143,66 & 160,10 & 133,54 \\
\hline
\end{tabular}

B: bovinos

mayores diferencias climáticas, pero se tiene evidencia que las temperaturas ambientales elevadas son factor predisponente para la infección, así como el estrés térmico y el manejo inadecuado (Wouda et al. 1999b). Habría que considerar aspectos de manejo intrínsecos para el control de la enfermedad, tales como la eliminación de placentas, que puedan infectar al hospedero definitivo, la limitación del acceso de los perros al alimento del ganado, así como el libre tránsito de animales, que pudieran ser portadores de la enfermedad. En este estudio, la edad no fue un factor de riesgo de importancia en la presentación de la enfermedad, lo que concuerda con Patitucci et al. (1999), quienes expresan que las vacas de cualquier edad son susceptibles de neosporosis.

Se determinó una mayor frecuencia de infección en animales doble propósito en comparación con el ganado de carne; no obstante, la asociación de los resultados no reveló diferencias estadísticas significativas en la variable tipo de explotación con $N$. caninum $\left(\mathrm{X}^{2}: 0,507, \mathrm{p} \geq 0,05\right)$, como se puede evidenciar en la tabla 2.

Tabla 2. Seropositividad a $N$. caninum y nivel de sarcocystina en bovinos, del municipio de Montería, según el propósito productivo.

\begin{tabular}{|c|c|c|}
\hline Indicadores & Doble propósito & Carne \\
\hline Seroprevalancia (\%) & $80,00(52 / 162)$ & $71,70(110 / 162)$ \\
\hline Nivel de sarcocystina UI/L & 143,06 & 136,87 \\
\hline
\end{tabular}

La mayor frecuencia de infección en animales doble propósito, en comparación con el ganado de carne, podría ser explicada por mayores densidades de población en las primeras, lo que favorece la diseminación de la enfermedad, debida a las condiciones de estrés ocasionadas por este tipo de manejo, aunque no hubo asociación entre la presentación de $N$. caninum en relación con el propósito zootécnico o el tipo de explotación, además, de que existen evidencias de que la neosporosis en ganado de carne es mucho más baja, que en ganado de leche (Moore et al. 2001).
En la tabla 3, se observa que la seroprevalencia y el nivel de sarcocystina en vacas, tanto repetidoras como en las que presentaron aborto, no mostraron diferencias estadísticas significativas; no obstante, se debe destacar que la seroprevalencia fue mayor en vacas con aborto, debido a que existe una relación directa entre los niveles de toxina en sangre y desórdenes generales en los caninos, que actúan como huésped definitivo del parásito (Pulido et al. 2009).

Tabla 3. Seropositividad a $N$. caninum y nivel de sarcocystina en hembras bovinas, del municipio de Montería, según el estado reproductivo.

\begin{tabular}{|c|c|c|}
\hline Indicadores & Aborto & Repetidoras \\
\hline Seroprevalencia (\%) & $71,2(52 / 73)$ & $77(47 / 61)$ \\
\hline Nivel de sarcocystina UI/L & 141,02 & 151,88 \\
\hline
\end{tabular}


El 45,1\% (73/134) hembras bovinas, registraron historia de aborto, donde el $71,2 \%$ (52/73) fueron positivas a N. caninum; entre tanto, el 37,7\% (61/134) de las hembras del estudio, se reportaron con repetición de servicios, de las cuales, el $77 \%$ (47/61) presentaron seropositividad al parasito. Se debe tener en cuenta que las repeticiones de servicio pueden ser a la interrupción de la gestación o porque no hubo fecundación. Se desconoce si $N$. caninum ocasiona pérdidas tempranas de preñez; sin embargo, sí se ha reportado la presencia de abortos en el ganado infectado con $N$. caninum (Hoar et al. 1996; Williams et al. 1997; Waldner et al. 1998).

En cuanto al feto, puede morir en el útero, momificado, autolisado, nacer muerto, nacer vivo y morir inmediatamente o nacer clínicamente normal, pero crónicamente infectado (Daft et al. 1996; Zambrano et al. 2001). Baillargeon et al. (2001) y Landmann et al. (2002) demostraron que embriones de donantes positivas, cuando se transfiere a los receptores negativos, el ciclo de la infección congénita se rompe, pero cuando las receptoras son positivas, el feto se infecta, independientemente de la condición de las donantes.
La monta natural fue una práctica común en esta región, que llega a ser un factor de riesgo. La alta seropositividad a anticuerpos de $N$. caninum en los toros, 78,6\% (22/28), podría ser explicada por un mayor número de contactos sexuales con hembras seropositivas (Björkman et al. 2000); asimismo, Ortega-Mora et al. (2003) expresaron que la transmisión venérea del parásito podría ser posible, ya que describieron la presencia esporádica de ADN de $N$. caninum, tanto en semen fresco como congelado, por lo que, debido a que los toros que presentaron sero-reactividad son portadores del parasito y posee la capacidad de diseminarlo; sin embargo, no hubo evaluación y aislamiento en semen de los toros positivos.

La seroprevalencia en los animales de siete o más años fue superior que los animales más jóvenes de 2 a 6 años, similares a las concentraciones de sarcocystina, aunque sin diferencias estadísticas significativas en animales más viejos, de siete o más años, como se puede analizar en la tabla 4.

Tabla 4. Seropositividad a N. caninum y nivel de sarcocystina en bovinos, del municipio de Montería, según la edad.

\begin{tabular}{|c|c|c|}
\hline Indicadores & Dos a seis años & siete o más \\
\hline Seroprevalancia (\%) & $32,1(52 / 162)$ & $42,7(69 / 162)$ \\
\hline Nivel de sarcocystina UI/L & 151,21 & 134,42 \\
\hline
\end{tabular}

No fue posible determinar -como factor de riesgo-, la presencia de caninos en las fincas y la seropositividad a $N$ caninum, porque solo fue muestreado un perro por cada una de las fincas involucradas en el estudio. De los 22 caninos analizados, el 50\% resultó positivo, lo que indica una alta presencia de infección en estos animales; sin embargo, la presencia de infección por $N$. caninum en estos perros que conviven con los bovinos en las fincas analizadas, abre la posibilidad de transmisión horizontal. Por lo tanto, el control de la circulación de perros en las fincas es una medida que se debe implementar, ya que existe una estrecha relación entre bovinos y perros criados en establos (Del Campo et al. 2003) y se ha demostrado que la proporción de bovinos seropositivos aumenta cuando existen perros en las fincas (Wouda et al. 1999a).

El aumento en el título de sarcocystina en animales adultos hace pensar que existe, posiblemente, una reinfección relacionada con factores estresantes $\mathrm{u}$ otras enfermedades virales que causen inmunosupresión, lo que podría estar facilitando el ingreso o reactivación de la $N$. caninum, en bovinos de la región. De acuerdo con lo expresado por Azumendi et al. (1995), quienes explican que los niveles de sarcocystina aumentan cuando los quistes parasitarios se rompen, ya que estos contienen millares de esporas que están suspendidas en un líquido compuesto, principalmente, por toxina del parásito, llamada sarcocystina, como podría suceder en los estados de replicación y multiplicación del parásito.

Se ha sugerido que la infección simultánea con el virus de la Diarrea Viral Bovina (BVD) o la inmunosupresión por la ingestión prolongada de micotoxinas podrían actuar como factores desencadenantes del aborto, debido a $N$. caninum en una explotación enzóoticamente infectada (Björkman et al. 2000).

La información seroepidemiológica de $N$. caninum sugiere que es necesario llevar a cabo estudios adicionales de seguimiento clínico y reproductivo de los animales 
seropositivos y los hatos donde ellos se encuentran, con el fin de determinar el posible impacto de este agente sobre los índices reproductivos, producción y la salud animal en las ganaderías.

Conflictos de intereses: El manuscrito fue preparado y revisado con la participación de todos los autores, quienes declaramos que no existe conflicto de intereses que ponga en riesgo la validez de los resultados presentados.

\section{BIBLIOGRAFÍA}

1. AZUMENDI, J.; GRANADA, I.; PINZÓN, C; REY, Y; FORERO, L; ESPITIA, S; GIL, M; AVELLO, $N$; ESGUERRA, A. 1995. Efectos de la toxina de Sarcocystis. Rev. Salud Animal. Cuba. 7(1):273-284.

2. BAILLARGEON, P.; FECTEAU, G.; PARÉ, J; LAMOTHE, P.; SAUVEÉ, R. 2001. Evaluation of the embryo transfer procedure proposed by the International Embryo Transfer Society as a method of controlling vertical transmission of Neospora caninum in cattle. J. Am. Vet. Med. Assoc. 218:1803-1806.

3. BJÖRKMAN, C.; ALENIUS, S.; EMANUELSSON, U.; UGGLA, A. 2000. Neospora caninum and bovine virus diarrhoea virus infections in Swedish dairy cows in relation to abortion. Vet. J. 159:201-206.

4. CAMPERO, C.M.; ANDERSON, M.L; CONOSCIUTO, G.; ODRIOZOLA, H.; BRETSCHNEIDER, G.; POSO, M.A. 1998 Neospora caninum associated abortion in dairy herd in Argentina. Vet. Rec.143:228-229.

5. DAFT, B.; BARR, B.; COLLINS, N.; SVERLOW, K. 1996. Neospora encephalomyelitis and polyradiculoneuritis in an aged mare with Cushing's disease. Eq. Vet. J. 29:240-243.

6. DAVISON, H.; FRENCH N.; TREES A. 1999. Herdspecific and age-specific seroprevalence of Neospora caninum. 14 British dairy herds. Vet. Rec. 144:547550.

7. DEL CAMPO, J.; CHÁVEZ, A.; DELGADO, A.; FALCÓN, N.; ORNELAS, A.; CASAS, E.; SERRANO, E. 2003. Frecuencia de Neospora caninum en perros de establos lecheros del Valle de Lima. Rev. Inv. Vet. Perú. 14:145-149.

8. DIJKSTRA, T.; BARKEMA, W.; EYSKER, M.; WOUDA, W. 2001. Evidence of post-natal transmission of Neospora caninum in Dutch dairy herds. Int. J. Parasitol 31:209-215.
9. DUBEY, J. 1999a. Neosporosis a newly recognized protozoan disease similar to toxoplasmosis. Infect. Dis. Rev. 2:129-130.

10. DUBEY, J. 1999b. Recent advances in Neospora and neosporosis. Vet. Parasitol. 84:349-367.

11. DUBEY, J. 2003. Neosporosis in cattle. J. Parasitol 89 (Suppl): S42-S56.

12. DUBEY, J.; BUXTON, D.; WOUDA, W. 2006. Pathogenesis of bovine neosporosis. J. Comp. Pathol. 134:267289.

13. DUBEY, J.; SCHARES, G.; ORTEGA-MORA, L. 2007. Epidemiology and control of neosporosis and Neospora caninum. Clin. Microbiol. Rev. 20:323367.

14. GARCÍA, F.; LISTA, D. 2005. Neosporosis y Tricomoniasis. Manual de ganadería de doble propósito. Facultad de Ciencias Veterinarias, Universidad del Zulia. p.323327.

15. GUEDES, M.; GUIMARÃES, A.; ROCHA, C.; HIRSC, C. 2008. Frequência de anticorpos anti-Neospora caninum em vacas e fetos provenientes de municípios do sul de Minas Gerais. Rev. Bras. Parasitol. Vet. 17(4):189-194.

16. GRANADOS, S.; RIVERA, H.; CASAS, E.; SUÁREZ, F.; ARANA, C.; CHÁVEZ, A. 2014. Seroprevalencia de Neospora caninum en bovinos lecheros de cuatro distritos del Valle del Mantaro, Junín. Rev. Inv. Vet. Perú. 25(1):58-64.

17. HOAR, B.; RIBBLE, C.; SPITZER, C.; SPITZER, P.; JANZEN, E. 1996. Investigation of pregnancy losses in beef cattle herds associated with Neospora sp. infection. Can. Vet. J. 37:364-366.

18. LANDMANN, J.; JILLELLA, D.; O'DONOGHUE, P.; McGOWAN, M. 2002. Confirmation of the prevention of vertical transmission of Neospora caninum in cattle by the use of embryo transfer. Aust. Vet. J. 80:502-503.

19. LÓPEZ, G.; RESTREPO, B.; RESTREPO, M.; LOTERO, M.; MURILLO, V.; CHICA, A.; CANO, J.; GIRALDO, J. 2007. Study to demonstrate the presence of Neospora caninum in bovines of the property San Pedro in the Fredonia municipality. Rev. CES. Med. Vet. Zootec 2(1):7-20. 
20. McALLISTER, M.; BJÖRKMAN, C.; ANDERSONSPRECHER, R.; ROGERS, D. 2000. Evidence of point-source exposure to Neospora caninum and protective immunity in a herd of beef cows. J. Am. Vet. Med. Assoc. 217:881-887.

21. MOORE, D.; ODEON, A.; CAMPERO, C. 2001. Neosporosis bovina, una actualización. Vet. Arg. 18(180):752-775.

22. MOORE, D.; CAMPERO, C.; ODEÓN, A.; POSO, M.; CANO, D.; LEUNDA, M. 2002. Seroepidemiology of beef and dairy herds and fetal study of Neospora caninum in Argentina. Vet. Parasitol. 107:303-316.

23. MOORE D.; DRAGHI, M.; CAMPERO, C.; CETRÁ, B.; ODEON, A.; ALCARAZ, E.; SPÄTH, E. 2003. Serological evidence of Neospora caninum infections in beef bulls in six counties of the Corrientes province, Argentina. Vet. Parasitol. 114(4):247-252.

24. MOORE, D. 2004. Neosporosis en South America. Fundación Antorchas. Chile. p.76-88.

25. MRAD, A. 2006. Ética en la investigación con modelos animales experimentales. Alternativas y las 3 RS de Russel. Una responsabilidad y un compromiso ético que nos compete a todos. Rev. Col. Bioética. 1(1):163-184.

26. MOURA, A.; SOUZA, A.; SARTOR, A.; BELLATO, V.; TEIXEIRA, E. 2012. Neospora caninum antibodies in dairy cattle of Lages Municipality, Santa Catarina State, Brazil. Arch. Med. Vet. 44:117-122.

27. OBANDO, C.; BRACAMONTE, M.; MONTOYA, A.; CADENAS, V. 2010. Neospora caninum en un rebaño lechero y su asociación con el aborto. Rev. Cient. FVC-LUZ. 20(3):235-239.

28. ORTEGA-MORA, L.; FERNANDEZ-GARCIA, A; GOMEZ-BAUTISTA, M. 2006. Diagnosis of bovine neosporosis: Recent advances and perspectivas. Acta Parasitol. 51(1):1-14.

29. ORTEGA-MORA, L.; FERRE, I.; DELPOZO, I.; CAETANODA SILVA, A.; COLLANTES-FERNÁNDEZ, E.; REGIDOR-CERRILLO, J. 2003. Detection of Neospora caninum in semen of bulls. Vet. Parasitol. 117:301-308.

30. OVIEDO, T.; BETANCUR, C.; MESTRA, A.; GONZÁLEZ, M.; REZA, L.; CALONGE, K. 2007. Estudio serológico sobre neosporosis en bovinos con problemas reproductivos en Montería, Córdoba, Colombia. Rev. MVZ Córdoba. 12(1):929-933.

31. PABÓN, J.; ESLAVA, J.; GÓMEZ, R. 2001. Generalidades de la distribución espacial y temporal de la temperatura del aire y de la precipitación en Colombia. Meteorol. Col. 4:47-59.

32. PATITUCCI, A.; PEREZ, M.; LÜDERS, C.; RATTO, M.; DUMONT, A. 1999. Evidencia serológica de infección por Neospora caninum en rebaños lecheros del sur de Chile. Arch. Med. Vet. 31:215-218.

33. PATITUCCI, A.; PEREZ, M.; ISRAEL, K.; ROZAS, M. 2000. Prevalencia de anticuerpos séricos contra Neospora caninum en dos rebaños lecheros de la IX Región de Chile. Arch. Med. Vet. 32(2):209-214.

34. PULIDO, M.; AZUMENDI, J.; PULIDO, A. 2009. Análisis clínico y paraclínico de caninos contaminados experimentalmente con Sarcocystis sp. Rev Colomb Cienc Pec. 22(3):549-550.

35. ICA-FEDEGAN. 2012. Resolución 002341. Instituto Colombiano Agropecuario. Bogotá, Colombia. URL Disponible desde Internet en: http://www.slideshare. net/Fedegan/resolucion-2341 (con acceso 20/02/2013).

36. SCHARES, G.; BARWALD, A.; STAUBACH, C.; SÖNDGEN, P.; RAUSER, M.; SCHRODER, R. 2002. p38-avidity-ELISA: examination of herds experiencing epidemic or endemic Neospora caninum-associated bovine abortion. Vet. Parasitol. 106:293-305.

37. SILVA, P.; CHÁVEZ, A.; RIVERA, H.; CASAS, E. 2002. Seroprevalencia de Neospora caninum en bovinos lecheros del valle de Lima. Rev. Inv. Vet. Perú 13(2):51-55.

38. SOFTWARE ESTADÍSTICO SPSS Inc. 2007. SPSS for Windows Version 16.0. Chicago, Illinois, USA: SPSS Inc.

39. SUÁREZ, C.; MALDONADO, J. 2012. Seropositividad a Neospora caninum en unidades de producción bovina del estado Lara, Venezuela. Zootecnia Trop. 30(1):35-41.

40. TREES, A.; WILLIAMS, D. 2005. Endogenous and exogenous transplacental infection in Neospora caninum and Toxoplasma gondii. Trends Parasitol. 21:558-561. 
41. VENTURINI, M.; VENTURINI, L.; BACIGALUPE, D.; MACHUCA, M.; ECHAIDE, I.; BASSO, W. 1999. Neospora caninum infections in bovine fetuses and dairy cows with abortions in Argentina. Int. J. Parasitol. 29:1705-1708.

42. WALDNER, C.; JANZEN, E.; RIBBLE, C. 1998. Determination of the association between Neospora caninum infection and reproductive performance in beef herds. J. Am. Vet. Med. Assoc. 213:685-690.

43. WILLIAMS, D.; McGARRY, J.; GUY, F.; BARBER, J.; TREES, A. 1997. Novel ELISA for detection of Neospora-specific antibodies in cattle. Vet. Rec 140:328-331.

44. WOUDA, W.; DIJKSTRA, T.; KRAMER, A.; VAN MAANEM, C.; BRINKHOF, J. 1999a. Seroepidemiological evidence for a relationship between Neospora caninum infections in dogs and cattle. Int. J. Parasitol. 29:1677-1682.

45. WOUDA, W.; BARTELS, C.; MOEN, A. 1999b. Caracteristics of Neospora caninum-associated abortion storms in dairy herds in The Netherlands (1995 - 1997). Theriogenology 52:233-245.

46. ZAMBRANO J.; COTRINO, V.; JIMÉNEZ C.; ROMERO, M.; GUERRERO, B. 2001. Evaluación serológica de Neospora caninum en bovinos en Colombia. Rev. Acovez. 26:5-10.

Recibido: Octubre 14 de 2014

Aceptado: Septiembre 26 de 2015

Cómo citar:

Cardona, J.A.; Martínez, Y.; Betancur, C.A. 2015. Seroepidemiología de hembras bovinas naturalmente infectadas por Neospora caninum en Córdoba, Colombia. Rev. U.D.C.A Act. \& Div. Cient. 18(2): 401-408. 